\title{
Some Soliton Solutions of Non Linear Partial Differential Equations by Tan-Cot Method
}

\author{
Raj Kumar ${ }^{1}$, Mukesh Kumar ${ }^{1}$ and Anshu Kumar ${ }^{2}$ \\ ${ }^{1}$ (Department of Mathematics, MNNIT Allahabad, Uttar Pradesh-211004, India) \\ ${ }^{2}$ (Department of Mathematics, Sharda University, Plot No. 32-34, Knowledge Park III, Greater Noida, \\ Uttar Pradesh-201306, India)
}

\begin{abstract}
Tan-cot method is applied to get exact soliton solutions of non-linear partial differential equations notably generalized Benjamin-Bona-Mahony, Zakharov-Kuznetsov Benjamin-Bona-Mahony, KadomtsovPetviashvilli Benjamin-Bona-Mahony and Korteweg-de Vries equations, which are important evolution equations with wide variety of physical applications. Elastic behavior and soliton fusion/fission is shown graphically and discussed physically as far as possible.
\end{abstract}

Keywords: Benjamin-Bona-Mahony equation, Tan-Cot method, soliton solutions.

\section{Introduction}

Authors considered the following non-linear partial differential equations:

$u_{t}+a u_{x}+b u^{2} u_{x}+\delta u_{x x t}=0 \quad$ Generalised Benjamin-Bona-Mahony

$u_{t}+u_{x}+a u\left(u^{2}\right)_{x}-b\left(u_{x t}+u_{y y}\right)_{x}=0 \quad$ Zakharov-Kuznetsov Benjamin-Bona-Mahony

$u_{x t}+u_{x x}+2 a u^{2} u_{x x}+k u_{y y}+4 a u u_{x}^{2}+u_{x x x t}=0$ Kadomtsov-Petviashvilli Benjamin-Bona-Mahony (1.3)

$u_{t}+6 u u_{x}+u_{x x x}=0$, Korteweg-de Vries

where $a, b, \delta$ and $k$ are non-zero arbitrary constants. The equations (1.1)-(1.4) are abbreviated as GKBBM, ZKBBM, KPBBM and KdV respectively. These equations attracted to a diverse group of researchers in view of their wide applications in many fields of sciences such as fluid mechanics, plasma and solid state physics, optical fibers and other related issues of engineering.

The BBM equation was introduced first by Benjamin et al [1]. Furthermore, Zhang et al [2] investigated it as a regularized version of the KdV equation for shallow water waves. Wang et al [3] and Wang [4] studied non-linear, dispersive and dissipative effects of BBM equation. The study of Benjamin et al [1] follows that both the equations KdV and BBM are valid at the same level of approximation, but BBM equation does have some advantages over the KdV equation from the computational mathematics viewpoint. In certain theoretical investigations, the BBM equation is superior as a model for long waves, and the word "regularized" refers to the fact that, from the standpoint of existence and stability, the equation offers considerable technical advantages over the KdV equation.

Two dimensional generalizations of BBM equation i.e. (1.2) and (1.3) are given by ZakharovKuznetsov and Kadomtsov-Petviashvilli respectively, which can be studied pursuing the literature [5-8] and references therein. Further, the main mathematical difference between KdV and BBM models can be most readily appreciated by comparing the dispersion relation for the respective linearised equations $[9,10]$. As far as applications of BBM equation is concerned, it is used in the study of drift waves in plasma or the Rossby waves in rotating fluids. Other applications of $\mathrm{KdV}$ and BBM equations in semiconductor, optical devices can be found in the work of Zhang et al [2].

Getting inspiration from many applications of BBM, ZKBBM, KPBBM and KdV equations in real life problems, authors attained the solitory solutions of these equations. The term soliton coined by Zabusky and Kruskal [11] after their findings that waves like particles retained their shapes and velocities after interactions. The first published observation [12] of a solitary wave i.e. a single and localized wave was made by a naval architect John Scott Russel in 1834. Russel explored his experiences in his report to the British Association [13]. In order to understand the non-linear phenomena of equations (1.1) - (1.4) in a better way it is important to seek their more exact solutions. A variety of useful methods notably Algebraic method [14], Exp-function method [15], Adomian modified method [16], Inverse scattering method [17], Tanh function method [18], Variational method [19], Similarity transformation methods using Lie group theory [20], Homotopy perturbation method [21], Jacobi elliptic function expansion method [22], Hirota method [23], Backlünd transformations method [24, 25], F-expansion method [26], Differential transformations method [27], Darboux transformations [28], Balance method [29], Sine-cosine method [30] and Tan-Cot method [31] were applied to investigate the solutions of non-linear partial differential equations.

Authors have been motivated from these researches and applied the Tan-Cot method to obtain soliton solutions of the equations (1.1) - (1.4). The tan-cot method is a direct and effective algebraic method for 
handling many non-linear evolution equations. It is a good tool to solve non-linear partial differential equations with genuine non-linear dispersion where solitary patterns solutions are generated [31].

The goal of the present work is to get exact soliton solutions of GBBM, ZKBBM, KPBBM and KdV equations by using tan-cot method. Elastic behavior and soliton fusion/fission of these solutions discussed physically as far as possible.

\section{Methodology}

Consider non-linear partial differential equation in the form:

$H\left(u, u_{x}, u_{y}, u_{t}, u^{n} u_{x}, u_{x y}, u_{x t}, \ldots, u_{x x t}, \ldots\right)=0$,

where $\mathrm{u}(\mathrm{x}, \mathrm{y}, \mathrm{t})$ is the soliton solution of $(2.1), \mathrm{u}_{\mathrm{x}}$ and $\mathrm{u}_{\mathrm{t}}$ etc. are partial derivatives of $\mathrm{u}$ with respect to $\mathrm{x}$ and $\mathrm{t}$ respectively. The term $u^{n} u_{x}$ shows non-linearity in (2.1). Since equation (2.1) admits soliton solution. Hence, we can use the transformation:

$u(x, y, t)=f(X)$,

where $\mathrm{X}=\mathrm{x}+\mathrm{y}-\mathrm{ct}+\mathrm{d}, \mathrm{c}$ is the speed of the travelling wave and $\mathrm{d}$ is a constant. It follows:

$\frac{\partial u}{\partial x}=\frac{\partial u}{\partial y}=\frac{\partial f}{\partial x}, \frac{\partial^{2} u}{\partial x \partial t}=-c \frac{d^{2} f}{d x^{2}}=-c \overline{\bar{f}}$ etc.

Making use of (2.3) into (2.1) yields a non-linear ordinary differential equation (ODE):

$G\left(f, \bar{f}, f^{n} \bar{f}, \overline{\bar{f}}, \ldots\right)=0$

The equation (2.4) is then integrated as long as all terms involve derivatives of $f$, where we ignore the constant of integration (Since $f \rightarrow 0$ as $X \rightarrow \pm \infty$ ). To get solution of (2.1) by applying Tan-Cot method, the function $\mathrm{f}(\mathrm{X})$ can be expressed either in the following forms:

$f(X)=\alpha \tan ^{\beta}(\mu X),|\mu X| \leq \frac{\pi}{2}$

or $f(X)=\alpha \cot ^{\beta}(\mu X),|\mu X| \leq \frac{\pi}{2}$,

where $\alpha, \beta$ and $\mu$ (wave number) are the unknown parameters. The derivatives of (2.5a) are:

$\bar{f}(X)=\alpha \beta \mu\left[\tan ^{\beta-1}(\mu X)+\tan ^{\beta+1}(\mu X)\right]$,

$\overline{\bar{f}}(X)=\alpha \beta \mu^{2}\left[(\beta-1) \tan ^{\beta-2}(\mu X)+2 \beta \tan ^{\beta}(\mu X)+(\beta+1) \tan ^{\beta+2}(\mu X)\right]$,

$\overline{\bar{f}}(X)=\alpha \beta \mu^{3}\left[(\beta-2)(\beta-1) \tan ^{\beta-3}(\mu X)+\left(3 \beta^{2}-3 \beta+2\right) \tan ^{\beta-1}(\mu X)+\left(3 \beta^{2}+3 \beta+2\right) \tan ^{\beta+1}(\mu X)\right.$

$\left.+(\beta+2)(\beta+1) \tan ^{\beta+3}(\mu X)\right]$,

$\overline{\overline{\bar{f}}}(X)=\alpha \beta \mu^{4}\left[(\beta-3)(\beta-2)(\beta-1) \tan ^{\beta-4}(\mu X)+4(\beta-1)\left(\beta^{2}-2 \beta+2\right) \tan ^{\beta-2}(\mu X)\right.$

$+2 \beta\left(3 \beta^{2}++5\right) \tan ^{\beta}(\mu X)+4(\beta+1)\left(\beta^{2}+\beta+2\right) \tan ^{\beta+2}(\mu X)$

$\left.+(\beta+1)(\beta+2)(\beta+3) \tan ^{\beta+4}(\mu X)\right]$,

and so on.

Authors found all solutions considering the form $\alpha \tan ^{\beta}(\mu \mathrm{X})$. Therefore, derivatives are calculated intentionally only for this form.

We can obtain an equation in different powers of tangent functions substituting the values of derivatives from (2.6) - (2.9) into (2.4). Then we collect the coefficients of each pair of tangent functions with same exponent from (2.4), where each term has to vanish. Consequently, one can obtain a system of algebraic equations in unknown parameters $\mathrm{c}, \alpha, \beta$ and $\mu$. Solving this system, we can get the soliton solutions of partial differential equation (2.1) by substituting the values of these parameters in (2.5a).

\section{Benjamin-Bona-Mahony (BBM) equation}

\section{Results And Discussions}

Authors studied the BBM equation in the form (1.1). Taking $X=x-c t+d$ and applying the above procedure on (1.1), it leads to:

$(\mathrm{a}-\mathrm{c}) \overline{\mathrm{f}}+\mathrm{bf}^{2} \overline{\mathrm{f}}-\mathrm{c} \delta \overline{\overline{\mathrm{f}}}=0, \quad \mathrm{a} \neq \mathrm{c}$

Integrating (3.1.1) with respect to $\mathrm{X}$ and ignoring constant of integration, one can obtain:

$(\mathrm{a}-\mathrm{c}) \mathrm{f}+\frac{\mathrm{b}}{3} \mathrm{f}^{3}-\mathrm{c} \delta \overline{\overline{\mathrm{f}}}=0$,

Making use of (2.5a) and (2.7) into (3.1.2) gives:

$$
\begin{aligned}
& (\mathrm{a}-\mathrm{c}) \alpha \tan ^{\beta}(\mu \mathrm{X})+\frac{\mathrm{b}}{3} \alpha^{3} \tan ^{3 \beta}(\mu \mathrm{X})-\mathrm{c} \delta \alpha \beta(\beta-1) \mu^{2} \tan ^{\beta-2}(\mu \mathrm{X})-2 c \delta \alpha \beta^{2} \mu^{2} \tan ^{\beta}(\mu \mathrm{X}) \\
& \quad-c \delta \alpha \beta(\beta+1) \mu^{2} \tan ^{\beta+2}(\mu \mathrm{X})=0
\end{aligned}
$$

Equating the exponents of second and third term of tangent functions in the equation (3.1.3), then collecting the coefficients of the terms involved tangent functions of the same exponent, where each term has to vanish. We obtain the following system of algebraic equations:

$3 \beta=\beta-2 ; \frac{b}{3} \alpha^{2}-2 c \delta \mu^{2}=0 ; \mu^{2}=\frac{a-c}{2 c \delta}$ 
On solving the system (3.1.4), we obtain:

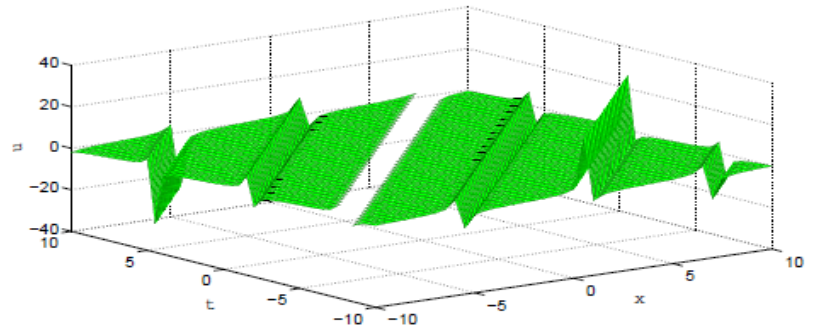

Figure 1: Multi soliton behaviour of $u(x, t)$ via expression (3.1.8).

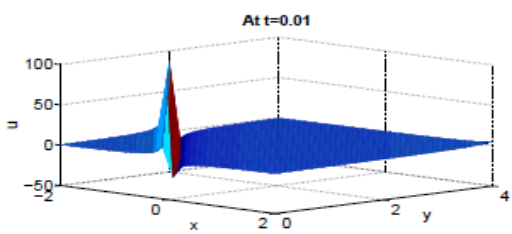

(a)

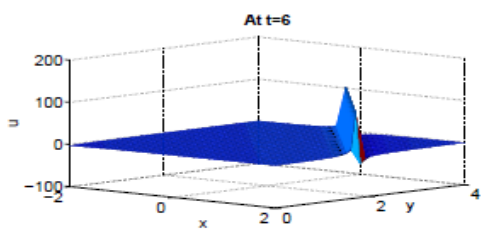

(b)

Figure 2: Soliton behaviour of $\mathbf{u}(\mathbf{x}, \mathbf{y}, \mathbf{t})$ via expression (3.2.9) for $-\mathbf{2} \leq \mathbf{x} \leq \mathbf{2 , 0} \leq \mathbf{y} \leq 4$

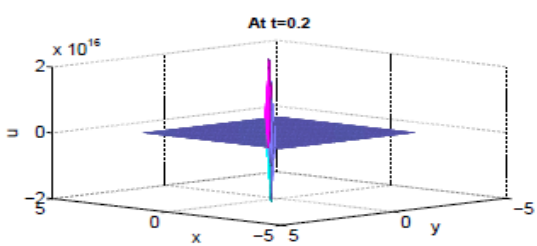

(a)

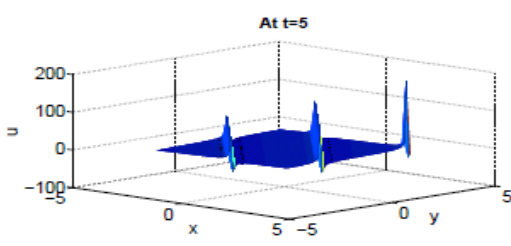

(b)

Figure 3: Soliton behaviour of $\mathbf{u}(\mathbf{x}, \mathbf{y}, \mathbf{t})$ via expression (3.3.9) for $-3 \leq x, y \leq 3$

$\beta=-1 ; \alpha=-\sqrt{\frac{3(a-c)}{b}} ; \mu=\sqrt{\frac{(a-c)}{2 c \delta}}$

It follows immediately:

$\frac{a-c}{b}>0$ and $\frac{a-c}{c \delta}>0$

Thus, soliton solution of BBM equation (1.1) is furnished by:

$u(x, t)=-\sqrt{\frac{3(a-c)}{b}} \cot \left[\sqrt{\frac{(a-c)}{2 c \delta}}(x-c t+d)\right], \quad$ where $\quad 0<|x-c t+d|<\frac{\pi \sqrt{c \delta}}{\sqrt{2(a-c)}}$

For $\mathrm{a}=\delta=\mathrm{d}=2, \mathrm{~b}=4$ and $\mathrm{c}=1$, equation (3.1.7) becomes:

$u(x, t)=-\frac{\sqrt{3}}{2} \cot \left[\frac{x-t+2}{2}\right]$

Evolutional profile of $u(x, t)$ through the expression (3.1.8) is shown in the figure 1 for the domain $-10 \leq$ $x, t \leq 10$. It is obvious from figure 1 that elastic behavior of multi solitons can be viewed.

\section{Zakharov-Kuznetsov-Benjamin-Bona-Mahony (ZKBBM) equation}

Authors found the soliton solutions of the ZKBBM governed by (1.2). Equation (1.2) can be rewritten in simplified form as:

$\mathrm{u}_{\mathrm{t}}+\mathrm{u}_{\mathrm{x}}+2 \mathrm{au}^{2} \mathrm{u}_{\mathrm{x}}-\mathrm{bu}_{\mathrm{xxt}}-\mathrm{bu}_{\mathrm{xyy}}=0$

The wave variable $\mathrm{X}=\mathrm{x}+\mathrm{y}-\mathrm{ct}+\mathrm{d}$ carries the equation (3.2.1) into an ODE represented by:

$(1-c) \bar{f}+2 a f^{2} \bar{f}-b(1-c) \overline{\bar{f}}=0, \quad c \neq 1$

Integrating (3.2.2) and ignoring the arbitrary constant of integration, one can get: 
$(1-c) f+\frac{2 a}{3} f^{3}-b(1-c) \overline{\bar{f}}=0$

Substituting the derivatives from equations (2.5) and (2.7) into (3.2.3), it provides us:

$$
\begin{aligned}
& \alpha(1-c) \tan ^{\beta}(\mu \mathrm{X})+\frac{2 \mathrm{a}}{3} \alpha^{3} \tan ^{3 \beta}(\mu \mathrm{X})-\mathrm{b}(1-c) \alpha \beta(\beta-1) \mu^{2} \tan ^{\beta-2}(\mu \mathrm{X})-2 \mathrm{~b}(1-c) \alpha \beta^{2} \mu^{2} \tan ^{\beta}(\mu \mathrm{X}) \\
& -b(1-c) \alpha \beta(\beta+1) \mu^{2} \tan ^{\beta+2}(\mu \mathrm{X})=0
\end{aligned}
$$

Equating the exponents of second and third tangent functions in the equation (3.2.4), and then we collect the coefficients of each pair of tangent functions of the same exponent, where each term has to vanish. Finally, we obtain the following system:

$3 \beta=\beta-2 ; 1-2 b \beta^{2} \mu^{2}=0 ; \frac{2 a}{3} \alpha^{2}-b(1-c) \beta(\beta-1) \mu^{2}=0$

Thus we obtain:

$\beta=-1 ; \alpha=-\sqrt{\frac{3(1-c)}{2 a}} ; \mu=\frac{1}{\sqrt{2 b}}$

It follows:

$b>0$ and $\frac{1-c}{a}>0$

In view of (3.2.6), solution of ZKBBM equation (1.2) is given by:

$\mathrm{u}(\mathrm{x}, \mathrm{y}, \mathrm{t})=-\sqrt{\frac{3(1-\mathrm{c})}{2 \mathrm{a}}} \cot \left[\frac{1}{\sqrt{2 \mathrm{~b}}}(\mathrm{x}+\mathrm{y}-\mathrm{ct}+\mathrm{d})\right], \quad$ with $\quad 0<|x+y-c t+d|<\frac{\pi \sqrt{b}}{\sqrt{2}}$

Setting arbitrary constants $\mathrm{a}=-2, \mathrm{~b}=\mathrm{c}=2$ and $\mathrm{d}=0$ in (3.2.8), it becomes:

$u(x, y, t)=-\frac{\sqrt{3}}{2} \cot \left[\frac{x+y-2 t}{2}\right]$

Solutions graphs via expression (3.2.9) are shown in the figure 2 for $-2 \leq \mathrm{x} \leq 2,0 \leq \mathrm{y} \leq 4$ at $\mathrm{t}=0.01$ and 6. In this spatial range, elastic single soliton can be observed in the figures 2(a) and 2(b).

\section{Kadomtsov-Petviashvilli-Benjamin-Bona-Mahony (KPBBM) equation}

Authors considered the KPBBM governed by (1.3). Applying the Tan-Cot method on (1.3) we can have following ODE:

$4 a f \overline{\mathrm{f}}^{2}+(1-\mathrm{c}+\mathrm{k}) \overline{\overline{\mathrm{f}}}+2 \mathrm{af}^{2} \overline{\overline{\mathrm{f}}}-\mathrm{c} \overline{\overline{\mathrm{f}}}=0$

Integrating (3.3.1) twice w.r.t. X (ignoring the arbitrary constants of integration), we obtain

$\frac{2 a}{3} f^{3}+(1-c+k) f-c \overline{\bar{f}}=0$,

Substituting the values of $f, \overline{\bar{f}}$ from of equations (2.5a) and (2.7) into (3.3.2), one can get

$(1-c+k) \alpha \tan ^{\beta}(\mu X)+\frac{2 a}{3} \alpha^{3} \tan ^{3 \beta}(\mu X)-c \alpha \beta(\beta-1) \mu^{2} \tan ^{\beta-2}(\mu X)-2 c \alpha \beta^{2} \mu^{2} \tan ^{\beta}(\mu X)-c \alpha \beta(\beta+$

1) $\mu^{2} \tan ^{\beta+2}(\mu \mathrm{X})=0$

Equating the exponents of second and third tangent functions in the equation (3.3.3), and then we collect the coefficients of each pair of tangent functions of the same exponent, where each term has to vanish. Finally, we obtain the following system:

$3 \beta=\beta-2 ; 1-c+k-2 c \beta^{2} \mu^{2}=0 ; \frac{2 \mathrm{a}}{3} \alpha^{2}-c \beta(\beta-1) \mu^{2}=0$

On solving the system (3.3.4): we get the following values of unknowns $\alpha, \beta$ and $\mu$;

$\beta=-1 ; \alpha=-\sqrt{\frac{3(1-c+k)}{2 a}} ; \mu=\sqrt{\frac{1-c+k}{2 c}}$

It follows immediately:

$\frac{1-c+k}{a}>0$ and $\frac{1-c+k}{c}>0$

In view of (3.3.5), solution of KPBBM equation (1.3) can be written in explicit manner as:

$u(x, y, t)=-\sqrt{\frac{3(1-c+k)}{2 a}} \cot \left[\sqrt{\frac{1-c+k}{2 c}}(x+y-c t+d)\right]$

where $0<|x+y-c t+d|<\frac{\pi \sqrt{c}}{\sqrt{2(1-c+k)}}$

For a $=1, \mathrm{c}=2, \mathrm{~d}=0, \mathrm{k}=4$, the equation (3.3.7) becomes:

$u(x, y, t)=-\frac{3}{\sqrt{2}} \cot \left[\frac{\sqrt{3}}{2}(x+y-2 t)\right]$

Evolutional profile for (3.3.9) is shown in the figure 3 for $-5 \leq x, y \leq 5$ at $t=0.2,5$. Fusion and fission of elastic solitons can be observed in the figures 3(a) and 3(b).

\section{Korteweg-de Vries(KdV) equation}

The KdV equation under our consideration is governed by (1.4). Using the same procedure as we have used in previous subsections to obtain an ODE: 
$-c \bar{f}+6 f \bar{f}+\overline{\bar{f}}=0$

Substituting the devatives of $f$ from (2.6) and (2.8) into (3.4.1), we have:

$-\operatorname{co} \beta \mu\left[\tan ^{\beta-1}(\mu \mathrm{X})+\tan ^{\beta+1}(\mu \mathrm{X})\right]+6 \alpha^{2} \beta \mu \tan ^{\beta}(\mu \mathrm{X})\left[\tan ^{\beta-1}(\mu \mathrm{X})+\tan ^{\beta+1}(\mu \mathrm{X})\right]$

$+\alpha \beta \mu^{3}\left[(\beta-1)(\beta-2) \tan ^{\beta-3}(\mu \mathrm{X})+\left(3 \beta^{2}-3 \beta+2\right) \tan ^{\beta-1}(\mu \mathrm{X})\right.$

$\left.+\left(3 \beta^{2}+3 \beta+2\right) \tan ^{\beta+1}(\mu \mathrm{X})+(\beta+1)(\beta+2) \tan ^{\beta+3}(\mu \mathrm{X})\right]=0$

The equation (3.4.2) is satisfied if the following system of algebraic equations holds:

$\beta=2 ; c=8 \mu^{2}, \alpha=-2 \mu^{2}$

By solving the system (3.4.3), one can find the following soliton solution of KdV equation (1.4):

$u(x, t)=\frac{c}{4}\left[1-\sec ^{2}\left\{\frac{\sqrt{c}}{2 \sqrt{2}}(x-c t+d)\right\}\right]$

For $\mathrm{c}=8, \mathrm{~d}=0$ equation (3.4.5) becomes:

$\mathrm{u}(\mathrm{x}, \mathrm{t})=2\left[1-\sec ^{2}(\mathrm{x}-4 \mathrm{t})\right]$

Evolutional profile for (3.4.5) is shown in the figure 4. Elastic multi solitons can be observed at regular intervals.

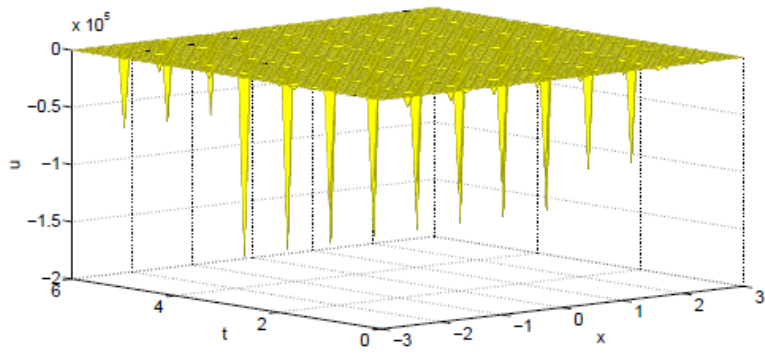

Figure 4: multi solitons solutions for (3.4.5) in domain $-3 \leq \mathrm{x} \leq \mathbf{3}, \mathbf{0} \leq \mathrm{t} \leq \mathbf{6}$.

\section{Conclusions}

In this work, the exact soliton solutions of generalised Benjamin-Bona-Mahony, Zakharov-KuznetsovBBM, Kadomtsov-Petviashvilli BBM, Korteweg-de Vries equation equations have been obtained successfully by using tan-cot method. The soliton behaviour of solutions with respect to time and space is shown and discussed physically wherever was possible. Authors are intend to get soliton solutions by applying this method to a system of non-linear partial differential equations like (2+1)-dimensional Boiti-Leon-Pempinelli (BLP) system.

\section{References}

[1] T. B. Benjamin, J. L. Bona, and J. J. Mahony, Model equations for long waves in non-linear dispersive systems, Philosophical Transactions of the Royal Society of London Series A, 272, 1972, 47-78.

[2] H. Zhang, G. M. Wei, and Y. T. Gao, On the general form of the Benjamin-Bona-Mahony equation in fluid mechanics, Czechoslovak Journal of Physics, 52, 2002, 373-377.

[3] B. Wang, and W. Yang, Finite-dimensional behaviour for the Benjamin-Bona-Mahony equation, Journal of Physics A: Mathematical and General, 30, 1997, 4877-4885.

[4] B. Wang, Regularity of attractors for the Benjamin-Bona-Mahony equation, Journal of Physics A: Mathematical and General, 31, $1998,7635-7645$.

[5] A. M. Wazwaz, Exact solutions of compact and noncompact structures for the KP-BBM equation, Applied Mathematics and Computation, 169, 2005, 700-712.

[6] A. M. Wazwaz, The tanh and the sine-cosine methods for a reliable treatment of the modied equal width equation and its variants, Communications in non-linear Science and Numerical Simulation, 11, 2006, 148-160.

[7] A. M. Wazwaz, Compact and noncompact physical structures for the ZKBBM equation, Applied Mathematics and Computation, 169, 2005, 713-725.

[8] M. A. Abdou, Exact periodic wave solutions to some non-linear evolution equations, Applied Mathematics and Computation, 6, 2008, 145-153.

[9] M. A. Karaca, and E. Hizel, Similarity reductions of Benjamin-Bona-Mahony equation, Applied Mathematical Sciences, 2, 2008, 463-469.

[10] D. J. Korteweg, and G. de Vries, on the change form of long waves advancing in a rectangular canal, and on a new type of long stationary waves, Philosophical Magazine, 39, 1895, 422-443.

[11] N. J. Zabusky, and M.D. Kruskal, Interaction of solitons in a collision less plasma and the recurrence of initial states, Physical Review Letters 15, 1965, 240-243.

[12] M. J. Ablowitz, and P. A. Clarkson, Solitons: non-linear evolution equations and inverse scattering (Cambridge UK, Cambridge University Press, 1991.)

[13] J. S. Russel, Report on waves, Rep. 14th Meet., British Association for the Advancement of Science (York, London, John Murray, 1844, 311-390.) 
[14] S. Zhang, and T. Xia, Further improved extended Fan sub-equation method and new exact solution of the (2+1) dimentional BroerKaup-Kupershmidt equations, Applied Mathematics and Computation, 182, 2006, 1651-1660.

[15] M. Kazeminia1, P. Tolou, J. Mahmoudi, I. Khatami, and N. Tolou, Solitary and periodic solutions of BBMB equation via Expfunction method, Advanced Studies in Theoretical Physics, 3, 2009, 461-471.

[16] S. Abbasbandy, and M. T. Darvishi, A numerical solution of Burgers' equation by modied Adomian method, Applied Mathematics and Computation, 163, 2005, 1265-1272.

[17] C. S. Gardner, J. M. Greene, M. D. Kruskal, and R. M. Miura, Method for solving the Korteweg-de Vries equation, Physical Review Letters, 19, 1967, 1095-1097.

[18] A. Soliman, The modied extended tanh-function method for solving Burgers type equations, Physica A, 361, 2006, $394-404$.

[19] J. H. He, Variational principles for some non-linear partial differential equations with variable coefficients, Chaos, Solitons and Fractals, 19, 2004, 847-851.

[20] P. J. Olver, Graduate texts in Mathematics: Applications of Lie groups to differential equations (New York, Springer-Verlag, 1993).

[21] J. H. He, Application of homotopy perturbation method to non-linear wave equations, Chaos, Solitons and Fractals, 26, 2005, 695700 .

[22] S. Liu, Z. Fu, S. Liu, and Q. Zhaos, Jacobi elliptic function expansion method and periodic wave solutions of non-linear wave equations, Physics Letters A, 289, 2001, 69-74.

[23] R. Hirota, Exact solution of the Korteweg-de Vries equation for multiple collisions of solitons, Physics Review Letters, 27, 1971, $1192-1194$.

[24] M. R. Miurs, Backlünd transformation (Berlin, Springer-Verlag, 1978).

[25] W. Hong, and Y. Jung, Auto-Backlünd transformation and analytic solutions for general variable-coefficient KdV equation, Physics Letters A, 257, 1999, 149-152.

[26] S. Zhang, Application of Exp-function method to a KdV equation with variable coecients, Physics Letters A, 365, 2007, 448-453.

[27] F. Ayaz, Solutions of the system of differential equations by differential transform method, Applied Mathematics and Computation, 147, 2004, 547-567.

[28] V. B. Matveev, and M. A. Salle, Darboux transformation and solitons (Berlin, Springer-Verlag, 1991).

[29] M. Wang, Exact solution for a compound KdV-Burgers equation, Physics Letters A, 213, 1996, $279-287$.

[30] C. Yan, A simple transformation for non-linear waves, Physics Letters A, 224, 1996, 77-84.

[31] A. J. M. Jawad, New exact solutions of non-linear partial differential equations using tan-cot function method, Studies in Mathematical Sciences, 5, 2012, 13-25. 